\title{
ON NORMAL STRUCTURE, FIXED POINT PROPERTY AND CONTRACTIONS OF TYPE $(\gamma)$
}

\author{
M. A. KHAMSI \\ (Communicated by William J. Davis)
}

\begin{abstract}
We prove that a Banach space $X$ has normal structure provided it contains a finite codimensional subspace $Y$ such that all spreading models for $Y$ have normal structure. We show that a Banach space $X$ is strictly convex if the set of fixed points of any nonexpansive map defined in any convex subset $C \subset X$ is convex and give a sufficient condition for uniform convexity of a space in terms of nonexpansive map of type $(\gamma)$.
\end{abstract}

\section{INTRODUCTION}

Our aim is to study the normal structure for Banach spaces. We recall that a normed space $X$ is said to have normal structure (N.S.) if and only if every convex bounded set in $X$ which contains more than one point has a nondiametral point.

This property was introduced by Brodskii and Milman [3] who also gave the following characterization in terms of sequences: a space $X$ has N.S. if and only if $X$ contains no bounded diametral sequence. For normal type structures and their applications to fixed point property, we refer to the exhaustive survey of Kirk [9].

The first theorem of this paper states that if $Y$ is a finite codimensional subspace of $X$ which has the $M$-normal structure, then $X$ has the normal structure. We also generalize a theorem of Zizler [13] on spaces which are uniformly convex in every direction and prove a converse to a theorem of Bruck [4] on nonexpansive mappings of type $(\gamma)$.

\section{DEFINITIONS AND NOTATIONS}

In this paper $X$ will always denote a real Banach space; $\operatorname{sp}(A)$ denotes the subspace generated by $A$ (subset of $X$ ) and we will say $A$ is finite dimensional if $\operatorname{sp}(A)$ is a finite dimensional subspace of $X . S(X)$ denotes the unit sphere of $X$. For terms not explicitly defined reference may be made to Day's book [5].

Received by the editors October 12, 1986 and, in revised form, September 12, 1988.

1980 Mathematics Subject Classification (1985 Revision). Primary 47H10.

Key words and phrases. Nonexpansive mappings, normal structure, fixed point property. 
Definitions. (1) A point $x$ of a convex bounded set $C$ is called a nondiametral point of $C$ iff $\sup _{y \in C}\|x-y\|<\operatorname{diam} C$.

(2) A sequence $\left(x_{n}\right)$ is called diametral if

$$
c=\operatorname{diam}\left(x_{i}\right)>0 \text { and } \lim _{n} d\left(x_{n+1}, \operatorname{conv}_{i \leq n}\left(x_{i}\right)\right)=c .
$$

It follows that

* For every $x \in \operatorname{conv}\left(x_{n}\right)$, we have: $\lim _{n}\left\|x_{n}-x\right\|=c$.

* If $\left(x_{n}\right)$ is diametral any subsequence of $\left(x_{n}\right)$ is also diametral and is not convergent. The diametral property is invariant by translation.

(3) We say that $X$ is uniformly convex in the direction $z \in S(X)$ if for each $\varepsilon>0$

$$
\delta(\varepsilon, z)=\inf \left\{1-\left\|\frac{x+y}{2}\right\| ;\|x\|=\|y\|=1 \text { and } x-y=\varepsilon z\right\}>0 .
$$

We say that $X$ is uniformly convex if $\delta_{X}(\varepsilon)=\inf \{\delta(\varepsilon, z) ; z \in S(X)\}>0$ for every $\varepsilon>0$.

(4) The ultrapower $\mathbf{X}$ of $X$ is defined by $\mathbf{X}=1_{\infty}(X) / \mathscr{K}$, where $1_{\infty}(X)=$ $\left\{\left(x_{n}\right) ; x_{n} \in X\right.$ and $\left.\operatorname{Sup}_{n}\left\|x_{n}\right\|<+\infty\right\}$ and $\mathscr{K}=\left\{\left(x_{n}\right) \in 1_{\infty}(X) ; \lim _{\mathscr{U}}\left\|x_{n}\right\|=\right.$ $0\}$ where $\mathscr{U}$ is a free ultrafilter over $N$.

Let $(\mathrm{P})$ be a property on $X$. We say that $X$ has the property super-(P) if every subspace of $\mathbf{X}$ has the property $(\mathrm{P})$. For more details about ultrapowers, we refer to [11].

(5) Let $\Gamma=\left\{\gamma: R_{+} \rightarrow R_{+} ; \gamma\right.$ continuous and $\gamma(r)=0$ iff $\left.r=0\right\}$. We say that $T: C \rightarrow X$ is a nonexpansive map if $\|T(x)-T(y)\| \leq\|x-y\|$ for every $x, y$ in $C$. The fixed point set of $T$ will be denoted by $F_{T}$. We will say that $T$ is of type $(\gamma)$ if there exists $\gamma$ in $\Gamma$ such that for every $x, y$ in $C$

$$
\gamma\left(\left\|T\left(\frac{x+y}{2}\right)-\frac{T(x)+T(y)}{2}\right\|\right) \leq\|x-y\|-\|T(x)-T(y)\| .
$$

Denote $\gamma(T)=\{\gamma \in \Gamma$; such that $(* *)$ holds $\}$.

(6) A Banach space $F$ is called a spreading model of $X$, generated by the bounded sequence $\left(x_{n}\right)$, if the following hold.

(a) $X \subset F$ and there is a sequence $\left(e_{n}\right)$ in $F$ with $F$ the closed linear span of $X \cup\left\{e_{n}, n \in N\right\}$

(b) There exists a subsequence $\left(y_{n}\right)$ of $\left(x_{n}\right)$ so that $\left(y_{n}\right)$ has no norm convergent subsequence and for all $x \in X, n \in N$ and scalars $\left(c_{i}\right)_{1 \leq i \leq n}$ we have

$$
\left\|x+\sum_{1}^{n} c_{i} e_{i}\right\|=\lim _{\substack{m_{1}<m_{2}^{2}<\cdots<m_{1} \rightarrow \infty \\ m_{1} \rightarrow \infty}}\left\|x+\sum_{1}^{n} c_{i} y_{m_{i}}\right\| .
$$

Let (P) be a property on $X$. We say that $X$ has the property $M-(\mathrm{P})$ if $X$ and every spreading model for $X$ has the property (P). For more information about spreading model and $M$-properties we refer to [2].

(7) Let $X$ and $Y$ be Banach spaces and consider the space $X \oplus Y$. A norm $N$ on $X \oplus Y$ is said to be of type (L) if: 
(i) The restrictions of $N$ to $X$ and $Y$ are the initial norms of $X$ and $Y$.

(ii) The natural projections associated to $X \oplus Y$ have norm 1 .

(iii) For every $x, x^{\prime}$ in $X$ and $y$ in $Y$ we have $N(x+y) \leq N\left(x^{\prime}+y\right)$ implies $\|x\| \leq\left\|x^{\prime}\right\|$.

\section{NORMAL STRUCTURE AND THE FIXED POINT PROPERTY}

It is well known that geometrical properties of finite codimensional subspaces provide for very little information about the normal structure properties of spaces. However we shall see below that some positive results can be obtained.

Theorem 1. Let $X$ be a Banach space, and $Y$ be a finite codimensional subspace of $X$. Suppose that $Y$ has $M$-normal structure. Then $X$ has normal structure. Proof. Suppose that $X$ fails to have normal structure. Then $X$ contains a diametral sequence $\left(x_{n}\right)$. Our assumptions on $Y$ imply that there exists a finite dimensional subspace $Z$ of $X$ such that $X=Z \oplus Y$. Then we can write $x_{n}=z_{n}+y_{n}$ with $y_{n}$ in $Y$ and $z_{n}$ in $Z$. Since $\left(x_{n}\right)$ is bounded, we deduce that $\left(z_{n}\right)$ has a convergent subsequence $\left(z_{n_{k}}\right)$. It follows that for every positive scalar $\left(c_{1}, \ldots, c_{k}\right)$ which verify $\sum_{1}^{k} c_{i}=1$, we have

$$
\lim _{n_{1}<\cdots<n_{k+1}}\left\|y_{n_{k+1} \rightarrow \infty}-\sum_{1}^{k} c_{i} y_{n_{i}}\right\|=\lim _{\substack{n_{1}<\cdots<n_{k+1} \\ n_{1} \rightarrow \infty}}\left\|x_{n_{k+1}}-z_{n_{k+1}}-\sum_{1}^{k} c_{i}\left(x_{n_{i}}-z_{n_{i}}\right)\right\| .
$$

But

and

$$
\lim _{\substack{n_{1}<\cdots<n_{k+1} \\ n_{1} \rightarrow \infty}}\left\|z_{n_{k+1}}-\sum_{1}^{k} c_{i} z_{n_{i}}\right\|=0
$$

Then

$$
\lim _{\substack{n_{1}<\cdots<n_{k+1} \\ n_{1} \rightarrow \infty}}\left\|x_{n_{k+1}}-\sum_{1}^{k} c_{i} x_{n_{1}}\right\|=\operatorname{diam}\left(x_{n}\right) \text {. }
$$

$$
\lim _{\substack{n_{1}<\cdots<n_{k+1} \\ n_{1} \rightarrow \infty}}\left\|y_{n_{k+1}}-\sum_{1}^{k} c_{i} y_{n_{i}}\right\|=c=\operatorname{diam}\left(x_{n}\right) \text {. }
$$

Let $F$ be the spreading model of $Y$, generated by $\left(y_{n_{k}}\right)$, and $\left(e_{i}\right)$ the sequence given by the definition 6 . We have

$$
\left\|e_{k+1}-\sum_{1}^{k} c_{i} e_{i}\right\|_{F}=\lim _{\substack{n_{1}<\cdots<n_{k+1} \\ n_{1} \rightarrow \infty}}\left\|y_{n_{k+1}}-\sum_{1}^{k} c_{i} y_{n_{t}}\right\|=c
$$

for every scalar $c_{1}, \ldots, c_{k}$ such that $\sum_{1}^{k} c_{i}=1$.

We conclude that $\left(e_{i}\right)$ is a diametral sequence in $F$. This implies that $F$ fails to have N.S. Contradiction.

Remark 1. If we refine the proof we can get the following. Suppose that $X=$ $Y \oplus Z$ where $Z$ is a Schur space and $Y$ has $M$-normal structure. Then every weakly compact convex subset $C$ has a nondiametral point.

Our next corollary can also be deduced from [7]. 
Corollary 1. Let $X$ be a Banach space, and $Y$ be a finite codimensional subspace of $X$. Suppose that $Y$ has super normal structure. Then $X$ has super normal structure.

Proof. It is enough to prove that any ultrapower of $X$ has N.S. Our hypothesis on $Y$ implies that there exists a continuous projection $P: X \rightarrow Y$. If we consider $\mathbf{X}$ and $\mathbf{Y}$ the ultrapowers of $X$ and $Y$, we define the canonical projection $\mathbf{P}: \mathbf{X} \rightarrow \mathbf{Y}$ by $\mathbf{P}\left(\left(x_{n}\right)\right)=\left(P\left(x_{n}\right)\right)$. It is obvious that the kernel of $\mathbf{P}$ is a finite dimensional subspace of $\mathbf{X}$. Then $\mathbf{Y}$ is a finite codimensional subspace of $\mathbf{X}$ which has the super normal structure. But it is well known [2] that every spreading model is isometric to a subspace of an ultrapower. Therefore according to Theorem $1, \mathbf{X}$ has normal structure.

Proposition 2. M-normal structure and super normal structure are not equivalent.

Proof. Consider $E_{n}=l_{1}^{n}$ and $X$ the $l_{2}$-sum of $\left(E_{n}\right)$. It is proved in [2] that all spreading models of $X$ are isometric to the $l_{2}$-sum of $X$ and $l_{2}$. In the other hand Landes [10] proved that $X$ has normal structure. Again by Landes' result we deduce that all the spreading models of $X$ have N.S. We conclude that $X$ has $M$-normal structure while every ultrapower $\mathbf{X}$ of $X$ contains $l_{1}$ and consequently fails to have normal structure.

The next result is a refinement of Zizler's theorem on uniform convexity in every direction.

Theorem 2. Let $X$ be a Banach space and $Y$ a finite codimensional subspace of $X$. Suppose that $X$ is uniformly convex in every direction $y \in S(Y)$. Then $X$ has normal structure.

Proof. Let $Q: X \rightarrow Z$ be a natural projection associated to the decomposition $X=Y \oplus Z$ (where $Z$ is a finite dimensional subspace of $X$ given by the assumption on $Y$ ) and $C$ be a bounded convex set of $X$, not reduced to a single point. Let us prove that $C$ contains a nondiametral point. Without loss of generality we can suppose that $0 \in C$. Let us assume first that there exist $x, y$ in $C$ such that $x \neq y$ and $Q(x)=Q(y)$. Then $x-y$ belongs to $Y$. Since $X$ is uniformly convex in every direction $z$ in $S(Y)$, we deduce that $1 / 2(x+y)$ is a nondiametral point of $C$. Assume now that $Q(x) \neq Q(y)$ for every $x \neq y$ in $C$. Then the restriction of $Q$ to $\operatorname{sp}(C)$ is one to one. Therefore $C$ is finite dimensional in $X$ and consequently it contains a nondiametral point (see [12]).

Example. Let $Y$ be a Banach space which is uniformly convex in every direction and $F$ be a Banach space. Then $X=F \oplus_{p} Y$, for $p \in[1, \infty[$, is uniformly convex in every direction $y \in S(Y)$.

We will say that $X$ has the fixed point property if for any nonempty weaklycompact convex subset $C$ and any nonexpansive mapping $T$ defined from $C$ into $C$, has a nonempty fixed point set. Recall the classical Kirk's theorem 
which says that $X$ has the fixed point property provided $X$ has N.S. Let us give the last theorem of this part.

Theorem 3. Let $X$ and $Y$ be Banach spaces which have the fixed point property. Consider on $X \oplus Y$ a norm of type $(L)$. Then for every weakly compact convex subsets $C_{1}$ and $C_{2}$ of $X$ and $Y$ we have $F_{T} \neq \varphi$ for every nonexpansive map $T: C_{1} \oplus C_{2} \rightarrow C_{1} \oplus C_{2}$.

Proof. Let $T: C_{1} \oplus C_{2} \rightarrow C_{1} \oplus C_{2}$ be a nonexpansive map. We can write $T=T_{1}+T_{2}$ with $T(x+y)=T_{1}(x+y)+T_{2}(x+y)$ for every $(x, y)$ in $C_{1} \times C_{2}$. Fix $x$ in $C_{1}$ and consider the map $T_{x}: C_{2} \rightarrow C_{2}$ defined by $T_{x}(y)=T_{2}(x+y)$ for every $y$ in $C_{2}$. The property (ii) in the definition of the norm of type (L) implies that $T_{x}$ is a nonexpansive map. Our hypothesis on $Y$ implies that the fixed point set of $T_{x}$ is nonempty. For every $x$ in $C_{1}$ let $y_{x}$ be a fixed point of $T_{x}$. Define $T_{0}: C_{1} \rightarrow C_{1}$ by $T_{0}(x)=T_{1}\left(x+y_{x}\right)$. It is clear that $T_{0}$ is nonexpansive. Indeed, $\left\|T\left(x+y_{x}\right)-T\left(x^{\prime}+y_{x^{\prime}}\right)\right\| \leq\left\|x+y_{x}-x^{\prime}-y_{x^{\prime}}\right\|$ but $T\left(x+y_{x}\right)=T_{0}(x)+y_{x}$ and $T\left(x^{\prime}+y_{x^{\prime}}\right)=T_{0}\left(x^{\prime}\right)+y_{x^{\prime}}$. Using the property (iii) we obtain $\left\|T_{0}(x)-T_{0}\left(x^{\prime}\right)\right\| \leq\left\|x-x^{\prime}\right\|$. Therefore the fixed point set of $T_{0}$ is nonempty since $X$ has the f.p.p. Let $x_{0}$ be a fixed point of $T_{0}$, then $T\left(x_{0}+y_{x_{0}}\right)=T_{0}\left(x_{0}\right)+y_{x_{0}}$. Therefore $T\left(x_{0}+y_{x_{0}}\right)=x_{0}+y_{x_{0}}$ which implies the conclusion of Theorem 3 .

Remarks. (1) We don't know if the conclusion of Theorem 3 holds for every convex weakly compact subset of $X \oplus Y$, even if $Y$ is a finite dimensional space.

(2) It is not hard to adapt the proof of Theorem 3 for metric spaces.

\section{A CHARACTERIZATION OF STRICT AND UNIFORM CONVEXITY}

In this section we give two main results. The first theorem states a characterization of strict convexity in terms of nonexpansive mappings. The proof can be seen as a refinement of DeMarr's argument [6]. First let us prove one technical lemma.

Lemma 2. Let $X$ be a Banach space, and $\left(\beta, \beta_{0}\right) \in R_{+} \times R_{+}^{*}$ be fixed numbers. Suppose that there exists $x$ and $y$ in $S(X)$ such that: $\|(x+y) / 2\|=1-\beta$ and $\|(x-y) / 2\|>\beta_{0}$ and $\beta<1 / 2$. Then for every $a, c$ in $[-1,1]$ one has,

$$
|c| \leq \frac{\beta_{0}+2 \beta}{\left.\left(1-\beta-2 \beta / \beta_{0}\right) \beta\right)_{0}}\left\|\frac{a(x-y)}{2}+\frac{c(x+y)}{2}\right\| .
$$

Proof. To simplify the computation we will denote $(x+y) / 2$ by $\vec{j}$ and $(x-y) / 2$ by $\vec{i}$. By Hann-Banach theorem there exists $x^{*}$ in $S\left(x^{*}\right)$ such that $x^{*}(\vec{j})=$ $1-\beta$. (\&) But $\left|x^{*}(x)\right| \leq 1$ and $\left|x^{*}(y)\right| \leq 1$. Using (\&) we deduce that $x^{*}(x)$ and $x^{*}(y)$ are positive. Therefore $x^{*}(x)=1-s$ and $x^{*}(y)=1-s^{\prime}$ with $s$ and $s^{\prime}$ positive which verify by $(\&) s+s^{\prime}=2 \beta$. Then $\left|x^{*}(\vec{j})\right|=$ $\left|s-s^{\prime}\right| / 2 \leq 2 \beta$ and $|a| \leq\left(1 / \beta_{0}\right)\|a \vec{i}\|$ for every $a$ in $R$. Let us prove our inequality. $|c|=\left|x^{*}(c \vec{j})\right| /(1-\beta) \leq\left|x^{*}(a \vec{i}+c \vec{j})\right| /(1-\beta)+\left|x^{*}(a \vec{i})\right| /(1-\beta)$. But 
$\left|x^{*}(a \vec{i})\right| \leq|a| 2 \beta \leq(|| a \vec{i}+c \vec{j} \|+|c|) 2 \beta / \beta_{0}$. Then $|c| \leq(1 / \beta-1)\left(1+2 \beta / \beta_{0}\right)|| a \vec{i}+$ $c \vec{j} \|+(1 /(1-\beta))\left(2 \beta / \beta_{0}\right)|c|$. This implies the desired inequality.

Theorem 4. Let $X$ be a Banach space. T.F.A.E.

(a) $X$ is strictly convex.

(b) Every nonexpansive map $T$ defined on a convex subset $C$, has a convex fixed point set.

Proof. It is well known that (a) implies (b). Let us prove that (b) implies (a). Suppose that $X$ is not strictly convex. Then there exist $x \neq y$ in $S(X)$ with $\|x+y\|=2$. Let $C=\left\{\beta(x+y) / 2+\beta^{\prime}(x-y) / 2 ;\left(\beta^{\prime},|\beta|\right) \in[0,1]\right\}$ and $T: C \rightarrow C$ defined by

$$
T\left(\beta\left(\frac{x+y}{2}\right)+\beta^{\prime}\left(\frac{x-y}{2}\right)\right)=\beta\left(\frac{x+y}{2}\right)-(1-|\beta|)\left(\frac{x-y}{2}\right) .
$$

Lemma 2 shows that $T$ is a nonexpansive mapping. The fixed points of $T$ are $z=\beta(x+y) / 2+(1-|\beta|)(x-y) / 2$ for every $\beta$ in $[-1,1]$. We conclude that $F_{T}$ is not convex. Which gives a contradiction with (b).

The notion of contractions of type $(\gamma)$ was introduced by Baillon [1]. Indeed he proved that any nonexpansive mapping defined on a bounded convex subset of $L_{p}$ for $p \in[1, \infty)$, is of type $(\gamma)$. Therefore he asked if someone can find a characterization of such spaces which have the same property as $L_{p}$. Bruck [4] give a partial answer in proving in that if $X$ is a uniformly convex space, then there exists $\gamma_{0} \in \Gamma$ such that for every closed bounded convex subset $C$, with diameter one, and $T: C \rightarrow X$ a nonexpansive map, then $T$ is of type $(\gamma)$ and $\gamma_{0} \in \gamma(T)$. Our main second theorem proves the converse to Bruck's result.

Theorem 5. Let $X$ be a Banach space. T.F.A.E.

(a) $X$ is uniformly convex.

(b) There exists $\gamma_{0} \in \Gamma$ such that every nonexpansive map $T$ defined on a convex subset $C$ in $X$, with diameter one, is of type $(\gamma)$ with $\gamma_{0} \in \gamma(T)$.

Proof. Bruck's theorem says exactly that (a) implies (b). Let us prove that (b) implies (a). Suppose that $X$ is not uniformly convex and that (b) holds. Then there exist $x_{n}, y_{n} \in S(X)$ and $\beta_{0}>0$ such that $\left\|x_{n}-y_{n}\right\| \geq \beta_{0}$ and $\left\|x_{n}+y_{n}\right\|=2\left(1-\beta_{n}\right) \rightarrow 2$ when $n$ tends to infinity $(* *)$. Put $\vec{i}_{n}=\left(y_{n}-x_{n}\right) / 2$ and $\vec{j}_{n}=\left(x_{n}+y_{n}\right) / 2$. Let $C_{n}=\left\{a \vec{i}_{n}+c \vec{j}_{n} ;(a,|c|) \in[0,1]\right\}$ and $T_{n}: C_{n} \rightarrow C_{n}$ be defined by $T_{n}\left(a \vec{i}_{n}+c \vec{j}_{n}\right)=(1-|c|) \vec{i}_{n}+c \vec{j}_{n}$. Lemma 2 shows that there exists $r_{n}>0$ such that for every $x, y$ in $C_{n}$ we have $\left\|T_{n}(x)-T_{n}(Y)\right\| \leq r_{n}\|x-y\|$. From $(* *)$ we deduce that $\lim _{n} r_{n}=1$. Consider $L_{n}=\left(1 / r_{n}\right) T_{n}$. Then $L_{n}$ is nonexpansive and is still defined on $C_{n}$. Therefore (b) implies that $L_{n}$ is of type $(\gamma)$ with $\gamma_{0} \in \gamma\left(L_{n}\right)$. This means that for every $x, y$ in $C_{n}$ we have

$$
(* * *) \quad \gamma_{0}\left(\left\|L_{n}\left(\frac{x+y}{2}\right)-\frac{1}{2}\left(L_{n}(x)+L_{n}(y)\right)\right\| \leq\|x-y\|-\left\|L_{n}(x)-L_{n}(y)\right\| .\right.
$$

Let $\mathbf{X}$ be an ultrapower of $X$ (see Definition 4). We define $\mathbf{C}$ and $\mathbf{T}$ canonically by $\mathbf{C}=\left\{\left(x_{n}\right) \in \mathbf{X} ; x_{n} \in C_{n}\right\}$ and $\mathbf{T}\left(x_{n}\right)=\left(T_{n}\left(x_{n}\right)\right)$. It is clear from 
$(* * *)$ that $\mathbf{T}$ is a nonexpansive map of type $(\gamma)$ with $\gamma_{0} \in \gamma(\mathbf{T})$. But $\mathbf{C}$ and $\mathbf{T}$ can be identified with the convex set and the nonexpansive map defined in the proof of the Lemma 2. Therefore the fixed point set of $\mathbf{T}$ is not convex and $\mathbf{T}$ cannot be of type $(\gamma)$. Contradiction.

Remark 2 . There exist a strictly convex Banach space $E$, a bounded convex subset $C$ and a nonexpansive map $T$ from $C$ into $C$ which has a nonempty fixed point set, but which is not of type $(\gamma)$ (See [8]).

Problem. Can we construct a Banach space $X$ which is not uniformly convex and where every nonexpansive mapping, defined on a bounded convex subset, if of type $(\gamma)$ ?

\section{REFERENCES}

1. B. Baillon, Comportement asymptotique des contractions et semi-groupes de contractionsEquation de Schrödinger nonlinéaires et divers, Thèses présentées à l'université Paris VI, 1978.

2. B. Beauzamy et J. T. Lapreste, Modèles étalés des espaces de Banach, Publication du Département de Mathématiques de l'Université Claude Bernard-Lyon I 1983.

3. M. S. Brodskii and D. P. Milman, On the center of a convex set, Dokl. Akad. Nauk USSR 59 (1948), 837-940.

4. R. Bruck, $A$ simple proof of the mean ergodic theorem for nonlinear contractions in Banach spaces, Israel J. Math. 32 (1979), 107-116.

5. M. M. Day, Normed linear spaces, 3rd ed., Springer-Verlag, 1973.

6. R. DeMarr, Common fixed points for comuuting contraction mappings, Pacific J. Math. (1963), 1139-1141.

7. J. R. Giles, B. Sims, and S. Swaminathan, A geometrically aberrant Banach space with normal structure, Bull. Austral. Math. Soc. 31 (1985), 121-124.

8. M. A. Khamsi, Étude de la propriéte du point fixe dans les espaces de Banach et les espaces metriques, Thèses présentées à l'Université Paris VI, 1987.

9. W. A. Kirk, Nonexpansive mappings and normal structure in Banach spaces, Proc. Research Workshop on Banach space theory, (B. L. Lin, ed.) University of Iowa, 1981.

10. T. Landes, Permanence properties of normal structure, Pacific J. Math. 110 (1984), 125-143.

11. B. Sims, "Ultra"-techniques in Banach space theory, Queen's University Kingston, Ontario, Canada.

12. S. Swaminathan, Normal structure in Banach spaces and its generalizations, Contemp. Math., volume 18, 1983, pp. 201-215, 1981.

13. V. Zizler, On some rotundity and smoothness properties of Banach spaces, Dissertationes Math. (Rozprawy Mat.) 87 (1971), 415-440.

Department of Mathematics, University of Southern California, los Angeles, CALIFORNiA 90089 